\title{
Buchbesprechung
}

\author{
W.J.B. Houston
}

\section{Walther's Orthodontic Notes}

1976. I74 S., 71 Abb., \& 3,75 (John Wright \& Sons, Bristol).

$\mathrm{Da}$ die vor 16 Jahren im Taschenbuchformat erschienenen Orthodontic Notes von Prof. Walther 3 Jahre nach seinem Tod durch W.J.B. Houston eine 3. überarbeitete Auflage erfahren haben, läßt auf eine weiterhin andauernde Beliebtheit dieses Büchleins bei englischen Studierenden schließen. Das konnte u. a. dadurch bedingt sein, daß diese ,Notizen“", hervorgegangen aus Walther's Vorlesungen am Royal Dental Hospital, School of Dental Surgery, London, in klarer, leicht verständlicher Sprache und übersichtlicher Form Grundkenntnisse vermitteln, die für die Praxis unbedingt notwendig sind.

Die Einteilung der Anomalien und ihre Besprechung erfolgt jeweils nach der Angle'schen Klassifikation. In der 3. Auflage sind zwei neue Kapitel hinzugekommen (in Zusammenarbeit mit Plint), in der ,,moderne Plattentechnik" in Kombination mit dem Headgear anhand guter, anschaulicher Abbildungen dargestellt wird; das verwundert zunächst, wird aber verständlich, da dieses Büchlein für Studenten gedacht ist und nach Ansicht des Herausgebers Multibandgeräte vorwiegend in die Hand des Facharztes gehören.

Die Kapitel über festsitzende und funktionelle Geräte sowie über chirurgische Kieferorthopädie (bearbeitet von M.J.C. Wake) und Lippen-Kiefer-Gaumenspalten (ebenfalls bearbeitet von D.A. Plint) wurden, wie der Herausgeber betont, mehr der Vollständigkeit halber eingefügt, um die Studenten auch mit dieser Problematik vertraut zu machen.

Wenn auch der Rahmen der bewußt beibehaltenen Notizensammlung durch die Aufnahme weiterer Literaturhinweise wohl kaum gesprengt worden wäre, sind die im Anhang angefügten Zusammenstellungen der hauptsächlichen Befunde sowie der wichtigsten Definitionen (einschließlich gnathologischer und cephalometrischer Begriffe) recht wertvoll.

Das handliche Büchlein vermittelt auf 170 Seiten erstaunlich viel kieferorthopädisches Wissen und trägt damit zum Verständnis der Probleme dieses Fachgebietes bei, wenngleich selbstverständlich - im Sinne der Notizensammlung - nicht alle kieferorthopädischen Aspekte umfassend dargestellt worden sind.

Der Preis ist gering. Die Ausstattung sehr gut, so daß auch deshalb dieses Büchlein englischsprechenden Studierenden sehr empfohlen werden kann. 